Теорія Ймовір. та Матем. Статист. Вип. 82, 2010
Theor. Probability and Math. Statist.

No. 82, 2011, Pages 103-114

S 0094-9000(2011)00830-7

Article electronically published on August 4, 2011

\title{
PROPERTIES OF INTEGRALS WITH RESPECT TO A GENERAL STOCHASTIC MEASURE IN A STOCHASTIC HEAT EQUATION
}

UDC 519.21

V. M. RADCHENKO

\begin{abstract}
We prove a theorem on the continuity with respect to a parameter and an analogue of Fubini's theorem for integrals with respect to a general stochastic measure defined on Borel subsets of $\mathbb{R}$. These results are applied to study the stochastic heat equation considered in a mild as well as in a weak form.
\end{abstract}

\section{INTRODUCTION}

Let $\mathbf{X}$ be an arbitrary set, let $\mathcal{B}$ be the $\sigma$-algebra of subsets of $\mathbf{X}$, and let $(\Omega, \mathcal{F}, \mathrm{P})$ be a complete probability space. By $L_{0}=L_{0}(\Omega, \mathcal{F}, \mathrm{P})$, we denote the set of all random variables (more precisely, the P-equivalent classes of random variables). The convergence in $L_{0}$ means the convergence in probability.

Definition 1.1. Every $\sigma$-additive mapping $\mu: \mathcal{B} \rightarrow L_{0}$ is called a stochastic measure on the $\sigma$-algebra $\mathcal{B}$.

The measure $\mu$ is not assumed to be nonnegative or adapted. In what follows the symbol $\mu$ denotes a stochastic measure on $\mathcal{B}$.

Below are some examples of stochastic measures.

1. If $X(x), a \leq x \leq b$, is a continuous square integrable martingale, then

$$
\mu(A)=\int_{a}^{b} \mathbf{1}_{A}(x) d X(x)
$$

is a stochastic measure defined on Borel subsets of $[a, b]$.

2. Similarly, the integral with respect to a fractional Brownian motion $B^{H}(x)$ with the Hurst index $H>1 / 2$ defines a stochastic measure (this follows from inequality (1.5) of [1]).

3. Other examples and conditions that increments of a stochastic process with independent increments generate a stochastic measure can be found in Sections 7 and 8 of [2].

An integral of the form $\int_{A} h(x) d \mu(x), A \in \mathcal{B}$, is constructed and its properties are studied in 3 for a measurable nonrandom function $h: \mathbf{X} \rightarrow \mathbb{R}$. The construction is standard and uses an approximation by simple functions. (A similar construction is presented in Section 7 of [2]; also see [4]). In particular, every measurable bounded function $h$ is integrable with respect to an arbitrary stochastic measure $\mu$. An analogue

2010 Mathematics Subject Classification. Primary 60G57, 60H15, $60 \mathrm{H} 05$.

Key words and phrases. Random measure, stochastic integral, stochastic Fubini theorem, stochastic convolution.

The research is supported by the Swedish Institute, grant SI-01424/2007.

(C)2011 American Mathematical Society 
of the Lebesgue dominated convergence theorem holds for this integral (see Corollary 1.2 in [3] or Proposition 7.1.1 in [2]).

An analogue of Fubini's theorem is proved in [5], and the continuity with respect to a parameter of an integral is obtained in [6] for stochastic measures $\mu$ defined on Borel subsets of the space $\mathrm{X}=[a, b]$. In [7] and [8], solutions of some partial differential equations are considered for the case where the randomness comes from an integral with respect to a stochastic measure $\mu$. In this paper, we generalize some of the results of [5, 6] to the case of $X=\mathbb{R}$ and find a relationship between weak and mild solutions of a stochastic heat equation.

We use the symbol $C$ throughout this paper to denote a constant that may be different in different equalities. Equalities and inequalities for random variables are understood to hold almost surely (unless we explicitly indicate that a relation is considered for every $\omega \in \Omega)$. The integrals of random functions with respect to real measures are considered for every $\omega \in \Omega$; the properties of such integrals can be found in 9 .

In Sections 2 and 3 of this paper, we prove some properties of integrals with respect to measures $\mu$ on the space $\mathbb{R}$. In Section 4 we consider the stochastic heat equation in the mild form and prove the equivalence of different forms of the integrals with respect to a stochastic measure. In Section [5, we show that a mild solution of the heat equation also is a weak equation.

In what follows we use the following result.

Lemma 1.1 ([8, Lemma 3.1], [10, Lemma 3.1]). Let $g_{l}: \mathrm{X} \rightarrow \mathbb{R}, l \geq 1$, be measurable functions such that the infinite sum $g(x)=\sum_{l=1}^{\infty}\left|g_{l}(x)\right|$ is finite and integrable with respect to $\mu$. Then

$$
\sum_{l=1}^{\infty}\left(\int_{\mathbf{X}} g_{l} d \mu\right)^{2}<\infty
$$

almost surely.

\section{The CONTINUITY ON $\mathbb{R}$ OF AN INTEGRAL With RESPECT TO A PARAMETER. StOChastic CONVOLUTION}

In this section, $\mathbf{X}=\mathbb{R}, \mathcal{B}$ is a Borel function, $\mathrm{T}$ is a metric space, and a function $f(x, t): \mathbb{R} \times \mathbf{T} \rightarrow \mathbb{R}$ is integrable in the variable $x$ with respect to the measure $\mu$ for every fixed $t \in \mathrm{T}$. Then one can consider the following random function:

$$
\zeta(t)=\int_{\mathbb{R}} f(x, t) d \mu(x), \quad t \in \mathrm{T} .
$$

Theorem 2.1. Let a function $f(x, \cdot)$ be continuous on $\mathrm{T}$ for every $x \in \mathbb{R}$. Assume that, for some $\gamma>1 / 2$,

$$
\left|f\left(x_{1}, t\right)-f\left(x_{2}, t\right)\right| \leq C\left|x_{1}-x_{2}\right|^{\gamma} .
$$

We further assume that $|x|^{\rho}$ is integrable on $\mathbb{R}$ with respect to $d \mu(x)$ for some $\rho>1 / 2$ and that $f$ is bounded, that is, $|f(x, t)| \leq C$. Then the random function $\zeta(t)$ defined by equality (11) has a version whose trajectories are continuous on $\mathrm{T}$.

Proof. We have

$$
\zeta(t)=\sum_{j \in \mathbb{Z}} \int_{(j, j+1]} f(x, t) d \mu(x),
$$


where the equality is understood almost surely and for every $t \in \mathrm{T}$. For all $j \in \mathbb{Z}$ and $n \geq 0$, we put

$$
\begin{gathered}
d_{k n}^{(j)}=j+k 2^{-n}, \quad 0 \leq k \leq 2^{n}, \quad \Delta_{k n}^{(j)}=\left(d_{(k-1) n}^{(j)}, \quad d_{k n}^{(j)}\right], \quad 1 \leq k \leq 2^{n}, \\
f_{n}^{(j)}(x, t)=\sum_{1 \leq k \leq 2^{n}} f\left(d_{(k-1) n}^{(j)}, t\right) \mathbf{1}_{\Delta_{k n}^{(j)}}(x), \quad j<x \leq j+1 .
\end{gathered}
$$

Condition (2) implies, in particular, that $f(\cdot, t)$ is continuous. Thus $f_{n}^{(j)}(x, t) \rightarrow f(x, t)$ as $n \rightarrow \infty$ for all $x \in(j, j+1]$ and $t$. The analogue of the Lebesgue theorem (see Corollary 1.2 in 3 ) implies that

$$
\int_{(j, j+1]} f_{n}^{(j)}(x, t) d \mu(x) \stackrel{\mathrm{P}}{\rightarrow} \int_{(j, j+1]} f(x, t) d \mu(x), \quad n \rightarrow \infty
$$

for all $t$.

Thus the sum

$$
\begin{aligned}
& \sum_{j \in \mathbb{Z}}\left(\int_{(j, j+1]} f_{0}^{(j)}(x, t) d \mu(x)\right. \\
& \left.\quad+\sum_{n \geq 1}\left(\int_{(j, j+1]} f_{n}^{(j)}(x, t) d \mu(x)-\int_{(j, j+1]} f_{n-1}^{(j)}(x, t) d \mu(x)\right)\right)
\end{aligned}
$$

is a version of the random function $\zeta(t)$. The terms

$$
\int_{(j, j+1]} f_{n}^{(j)}(x, t) d \mu(x)=\sum_{1 \leq k \leq 2^{n}} f\left(d_{(k-1) n}^{(j)}, t\right) \mu\left(\Delta_{k n}^{(j)}\right)
$$

are continuous functions with respect to $t$ and for all $\omega \in \Omega$ (this version of $\zeta(t)$ is considered below). We show that series (6) converges almost surely and uniformly in $\mathrm{T}$. Using (4), the Cauchy-Schwarz inequality, and bound (2) we obtain

$$
\begin{aligned}
\sum_{n \geq 1} \mid & \int_{(j, j+1]} f_{n}^{(j)}(x, t) d \mu(x)-\int_{(j, j+1]} f_{n-1}^{(j)}(x, t) d \mu(x) \mid \\
\leq & \sum_{n \geq 1} \sum_{1 \leq k \leq 2^{n}}\left|f\left(d_{(k-1) n}^{(j)}, t\right)-f\left(d_{\left(k^{\prime}-1\right)(n-1)}^{(j)}, t\right)\right|\left|\mu\left(\Delta_{k n}^{(j)}\right)\right| \\
\leq & \left\{\sum_{n \geq 1} \sum_{1 \leq k \leq 2^{n}} 2^{2 n \beta}\left|f\left(d_{(k-1) n}^{(j)}, t\right)-f\left(d_{\left(k^{\prime}-1\right)(n-1)}^{(j)}, t\right)\right|^{2}\right\}^{1 / 2} \\
& \times\left\{\sum_{n \geq 1} \sum_{1 \leq k \leq 2^{n}} 2^{-2 n \beta}\left|\mu\left(\Delta_{k n}^{(j)}\right)\right|^{2}\right\}^{1 / 2} \\
\leq & \left\{\sum_{n \geq 1} 2^{n(1+2 \beta-2 \gamma)}\right\}^{1 / 2}\left\{\sum_{n \geq 1} \sum_{1 \leq k \leq 2^{n}} 2^{-2 n \beta}\left|\mu\left(\Delta_{k n}^{(j)}\right)\right|^{2}\right\}^{1 / 2}
\end{aligned}
$$

for all $\beta>0$ and $\omega \in \Omega$. Here $k^{\prime}$ is chosen such that $\Delta_{k n}^{(j)} \subset \Delta_{k^{\prime}(n-1)}^{(j)}$; we fix some $0<\beta<\gamma-1 / 2$. 
Then the sum of absolute values of the terms of series (6) does not exceed

$$
\begin{aligned}
\sum_{j \in \mathbb{Z}}\left[|f(j, t) \mu((j, j+1])|+C\left\{\sum_{n \geq 1} \sum_{1 \leq k \leq 2^{n}} 2^{-2 n \beta}\left|\mu\left(\Delta_{k n}^{(j)}\right)\right|^{2}\right\}^{1 / 2}\right] \\
\leq C\left[\left(\sum_{j \in \mathbb{Z}}(|j|+1)^{2 \rho}(\mu((j, j+1]))^{2}\right)^{1 / 2}\left(\sum_{j \in \mathbb{Z}}(|j|+1)^{-2 \rho}\right)^{1 / 2}\right. \\
+\left(\sum_{n \geq 1} \sum_{j \in \mathbb{Z}} 2^{-2 n \beta}(|j|+1)^{2 \rho} \sum_{1 \leq k \leq 2^{n}}\left|\mu\left(\Delta_{k n}^{(j)}\right)\right|^{2}\right)^{1 / 2} \\
\left.\times\left(\sum_{j \in \mathbb{Z}}(|j|+1)^{-2 \rho}\right)^{1 / 2}\right] .
\end{aligned}
$$

For each $\rho>1 / 2$, we have $\sum_{j \in \mathbb{Z}}(|j|+1)^{-2 \rho}<+\infty$. The sums with stochastic measures are equal to

$$
\sum_{l=1}^{\infty}\left(\int_{\mathbf{X}} g_{l} d \mu\right)^{2}
$$

where $\left\{g_{l}(x), l \geq 1\right\}=\left\{(|j|+1)^{\rho} \mathbf{1}_{(j, j+1]}(x), j \in \mathbb{Z}\right\}$ for the first term on the right-hand side of (7), while

$$
\left\{g_{l}(x), l \geq 1\right\}=\left\{2^{-n \beta}(|j|+1)^{\rho} \mathbf{1}_{\Delta_{k n}^{(j)}}(x), j \in \mathbb{Z}, n \geq 1,1 \leq k \leq 2^{n}\right\}
$$

for the second one.

Since $|x|^{\rho}$ is integrable with respect to $d \mu(x)$, Lemma 1.1 implies convergence (15). The convergence is uniform, since the terms of the latter series do not depend on $t$.

A straightforward application of Theorem 2.1 allows one to prove the following result on the continuity of a convolution.

Corollary 2.1. Let $h(x): \mathbb{R} \rightarrow \mathbb{R}$ be a bounded function. Assume that, for some $\gamma>1 / 2$ and all $x_{1}$ and $x_{2}$,

$$
\left|h\left(x_{1}\right)-h\left(x_{2}\right)\right| \leq C\left|x_{1}-x_{2}\right|^{\gamma} .
$$

We further assume that $|x|^{\rho}$ is integrable on $\mathbb{R}$ with respect to $d \mu(x)$ for some $\rho>1 / 2$. Then there exists a version of the random function

$$
\zeta(t)=\int_{\mathbb{R}} h(t-x) d \mu(x), \quad t \in \mathbb{R},
$$

whose trajectories are continuous on $\mathbb{R}$.

Another result deals with the continuity of trajectories of a convolution represented as an integral over a finite interval.

Theorem 2.2. Let $h(x):[0, T] \rightarrow \mathbb{R}$ be a function such that $h(0)=0$. Assume that, for some $\gamma>1 / 2$ and all $x_{1}$ and $x_{2}$,

$$
\left|h\left(x_{1}\right)-h\left(x_{2}\right)\right| \leq C\left|x_{1}-x_{2}\right|^{\gamma} .
$$

Then there exists a version of the random function

$$
\zeta(t)=\int_{0}^{t} h(t-x) d \mu(x), \quad t \in[0, T],
$$

whose trajectories are continuous on $[0, T]$. 
Proof. We extend the definition of $h$ for $x<0$ by setting $h(x)=0$ for negative arguments. Then condition (8) holds for all $x \leq T$ and

$$
\zeta(t)=\int_{0}^{T} h(t-x) d \mu(x), \quad t \in[0, T] .
$$

It remains to apply Theorem 1 in [6] on the continuity of integrals dependent on a parameter (the integrals are considered over a finite interval). Another way to prove the same is to apply Theorem 2.1 for $\mu$ on $[0, T]$.

Note that the continuity of stochastic convolutions of the form (9) is studied in [1] for integrals with respect to semimartingales. The paper [11] contains a motivation for such a research, references to related results, and an example of a stochastic convolution that has no continuous version.

\section{Change of order of integration in $\mathbb{R}$}

Let $\left(\mathrm{Y}, \mathcal{B}_{\mathrm{Y}}\right)$ be a measurable space, $\mathrm{m}$ be a real finite nonnegative measure on $\mathcal{B}_{\mathrm{Y}}$, and let $\mathrm{Z}=\mathrm{X} \times \mathrm{Y}$ and $\mathcal{B}_{\mathrm{Z}}=\mathcal{B} \otimes \mathcal{B}_{\mathrm{Y}}$. A set $A \in \mathcal{B}$ is called $\mu$-negligible if $\mu(B)=0$ almost surely for all $B \subset A$ such that $B \in \mathcal{B}$.

The following result holds for all stochastic measures $\mu$ defined on an arbitrary measurable space $(\mathrm{X}, \mathcal{B})$.

Theorem 3.1 (Theorem 1 in [5]). There exists a unique stochastic measure $\eta$ defined on $\mathcal{B}_{\mathrm{Z}}$ such that $\eta\left(A_{1} \times A_{2}\right)=\mu\left(A_{1}\right) \mathrm{m}\left(A_{2}\right)$ for all $A_{1} \in \mathcal{B}$ and $A_{2} \in \mathcal{B}_{\mathrm{Y}}$.

If $f: Z \rightarrow \mathbb{R}$ is an integrable on $\mathbb{R}$ function with respect to $\eta$ for all fixed $x \in \mathbf{X}$ except a $\mu$-negligible set, then the function $f(x, \cdot): \mathrm{Y} \rightarrow \mathbb{R}$ is integrable on $\mathrm{Y}$ with respect to $\mathrm{m}$ and the function $\int_{Y} f(x, y) d \mathrm{~m}(y)$ is integrable on $\mathbf{X}$ with respect to $d \mu(x)$.

Moreover,

$$
\int_{\mathbf{Z}} f(x, y) d \eta=\int_{\mathbf{X}} d \mu(x) \int_{\mathbf{Y}} f(x, y) d \mathbf{m}(y) .
$$

The stochastic measure $\eta$ introduced in Theorem 3.1 (and which is the product of $\mu$ and $\mathrm{m}$ ) satisfies the following property.

Theorem 3.2 (Theorem 2 in [5]). Let $\mathrm{X}=[a, b] \subset \mathbb{R}$ and let $\mathcal{B}$ be a Borel set. Assume that $f: \mathbf{Z} \rightarrow \mathbb{R}$ is a measurable bounded function. Moreover let, for some $\gamma>1 / 2$ and all $x_{1}$ and $x_{2}$,

$$
\left|f\left(x_{1}, y\right)-f\left(x_{2}, y\right)\right| \leq C\left|x_{1}-x_{2}\right|^{\gamma} .
$$

Then

$$
\int_{\mathbf{Z}} f(x, y) d \eta=\int_{\mathbf{Y}} d \mathrm{~m}(y) \int_{\mathbf{X}} f(x, y) d \mu(x) .
$$

We generalize Theorem 3.2 by proving the following result for the stochastic measure $\eta$ introduced in Theorem 3.1 .

Theorem 3.3. Let $\mathbf{X}=\mathbb{R}$ and let $\mathcal{B}$ be a Borel set. Assume that $f: \mathbf{Z} \rightarrow \mathbb{R}$ is a measurable bounded function and that, for some $\gamma>1 / 2$,

$$
\left|f\left(x_{1}, y\right)-f\left(x_{2}, y\right)\right| \leq C\left|x_{1}-x_{2}\right|^{\gamma} .
$$
Then

$$
\int_{\mathbf{Z}} f(x, y) d \eta=\int_{\mathbf{Y}} d \mathrm{~m}(y) \int_{\mathbb{R}} f(x, y) d \mu(x) .
$$


Proof. Theorem 3.2 implies that

$$
\int_{(j, j+1] \times Y} f(x, y) d \eta_{j}=\int_{Y} d \mathrm{~m}(y) \int_{(j, j+1]} f(x, y) d \mu(x)
$$

for all $j \in \mathbb{Z}$, where $\eta_{j}$ is the product of the measures $\mu$ and $\mathrm{m}$ on $(j, j+1] \times \mathrm{Y}$. Since the measures $\eta_{j}$ and $\eta$ coincide on measurable multidimensional rectangles, we follow a standard reasoning and prove that the measures $\eta_{j}$ and $\eta$ coincide on the sets $(j, j+1] \times \mathrm{Y}$.

Consider

$$
\zeta(y)=\int_{\mathbb{R}} f(x, y) d \mu(x), \quad y \in \mathrm{Y} .
$$

Representation (6) holds for an appropriate version of this integral. Now we use the bound (7) for this representation with some random constant $K(\omega)$ and all $y \in \mathrm{Y}$ and $\omega \in \Omega$. Thus we obtain

$$
|\zeta(y)| \leq K(\omega)<+\infty .
$$

Summing up equalities (12) over all $j \in \mathbb{Z}$, applying the dominated convergence theorem for these integrals, and recalling the equality $\eta_{j}=\eta$, we prove (11).

\section{Stochastic heat Equation}

Consider the following equation:

$$
\left\{\begin{array}{l}
d u(x, t)=a^{2} \frac{\partial^{2} u(x, t)}{\partial x^{2}} d t+f(x, t, u(x, t)) d t+\sigma(x, t) d \mu(x), \\
u(x, 0)=u_{0}(x)
\end{array}\right.
$$

where $(x, t) \in \mathbb{R} \times[0, T], a>0$ is a constant, and $\mu$ is a stochastic measure defined on the Borel $\sigma$-algebra in $\mathbb{R}$.

The formal equality (13) is understood in the mild sense (14) or, in the case of $f=0$, in the weak sense (21)-(22) (see below).

Consider equation (13) in the mild sense:

$$
\begin{aligned}
u(x, t)= & \int_{\mathbb{R}} p(x-y, t) u_{0}(y) d y+\int_{0}^{t} d s \int_{\mathbb{R}} p(x-y, t-s) f(y, s, u(y, s)) d y \\
& +\int_{\mathbb{R}} d \mu(y) \int_{0}^{t} p(x-y, t-s) \sigma(y, s) d s .
\end{aligned}
$$

Here

$$
p(x, t)=\frac{1}{2 a \sqrt{\pi t}} e^{-|x|^{2} /\left(4 a^{2} t\right)}
$$

is the fundamental solution of the heat equation and

$$
u(x, t)=u(x, t, \omega): \mathbb{R} \times[0, T] \times \Omega \rightarrow \mathbb{R}
$$

is an unknown measurable random function (in other words, $u$ is a mild solution of equation (14)).

The existence and uniqueness of a solution of this equation is proved in 8 under some conditions. It is also proved in 8 that trajectories of a solution satisfy the Hölder condition.

In the rest of this paper we use the Besov spaces $B_{22}^{\alpha}([c, d])$. Recall that the norm in these classical spaces is defined for $0<\alpha<1$ as follows:

$$
\|g\|_{B_{22}^{\alpha}([c, d])}=\|g\|_{L_{2}([c, d])}+\left(\int_{0}^{d-c}\left(w_{2}(g, r)\right)^{2} r^{-2 \alpha-1} d r\right)^{1 / 2},
$$


where

$$
w_{2}(g, r)=\sup _{0 \leq h \leq r}\left(\int_{c}^{d-h}|g(y+h)-g(y)|^{2} d y\right)^{1 / 2} .
$$

The following result is needed to prove that the trajectories of the integral with respect to $\mu$ are bounded.

Lemma 4.1 ([8, Lemma 3.2]). Let $Z$ be an arbitrary set and let $q(y, z):[j, j+1] \times Z \rightarrow \mathbb{R}$ be a function such that $q(\cdot, z) \in B_{22}^{\alpha}([j, j+1])$ for some $1 / 2<\alpha<1$ and all $z \in Z$. Then there exists a version $\tilde{\zeta}(z)$ of the random function

$$
\zeta(z)=\int_{[j, j+1]} q(y, z) d \mu(y), \quad z \in Z
$$

such that

$$
\begin{aligned}
|\tilde{\zeta}(z)| \leq & |q(j, z) \mu([j, j+1])| \\
& +C\|q(\cdot, z)\|_{B_{22}^{\alpha}([j, j+1])}\left\{\sum_{n \geq 1} 2^{n(1-2 \alpha)} \sum_{1 \leq k \leq 2^{n}}\left|\mu\left(\Delta_{k n}^{(j)}\right)\right|^{2}\right\}^{1 / 2}
\end{aligned}
$$

for all $\omega \in \Omega$ and some constant $C$ that does not depend on $z$, $j$, and $\omega$.

In what follows we will assume the following conditions.

Condition 1. The function $\sigma(x, t): \mathbb{R} \times[0, T] \rightarrow \mathbb{R}$ is measurable and bounded.

Condition 2. For some $C>0$ and $\gamma(\sigma)>1 / 2$,

$$
\left|\sigma\left(x_{1}, t\right)-\sigma\left(x_{2}, t\right)\right| \leq C\left|x_{1}-x_{2}\right|^{\gamma(\sigma)} .
$$

When defining a mild solution of equation (13), the third term in (14) can be rewritten in several different ways. We show that, nevertheless, the third term of (14) will have the same value.

Corollary 4.1. Let $|y|^{\rho}$ be integrable on $\mathbb{R}$ with respect to $d \mu(y)$ for some $\rho>1 / 2$ and let a function $\sigma$ satisfy Conditions 1 and 2. Then

$$
\begin{aligned}
\int_{\mathbb{R}} d \mu(y) \int_{0}^{t} p(x-y, t-s) \sigma(y, s) d s & =\int_{0}^{t} d s \int_{\mathbb{R}} p(x-y, t-s) \sigma(y, s) d \mu(y) \\
& =\int_{\mathbb{R} \times[0, t]} p(x-y, t-s) \sigma(y, s) d \eta(y, s),
\end{aligned}
$$

where $\eta$ is the product of measures $\mu$ and ds on $\mathbb{R} \times[0, T]$. (Given $x$ and $t$, equality (17) holds almost surely.)

Proof. For an arbitrary set $A \in \mathcal{B}(\mathbb{R}) \otimes \mathcal{B}([0, t])$ and for a sequence of real numbers $t_{n} \uparrow t$, $t_{0}=0, t_{n}<t$, we derive from Theorem 3.1 that

$$
\begin{aligned}
\int_{\mathbb{R}} d \mu(y) \int_{0}^{t} p(x-y, t-s) \sigma(y, s) \mathbf{1}_{A}(y, s) \mathbf{1}_{\left(t_{n-1}, t_{n}\right]}(s) d s \\
\quad=\int_{\mathbb{R} \times[0, t]} p(x-y, t-s) \sigma(y, s) \mathbf{1}_{A}(y, s) \mathbf{1}_{\left(t_{n-1}, t_{n}\right]}(s) d \eta(y, s)
\end{aligned}
$$

(the integrand of the right-hand side of (18) is bounded and therefore is integrable with respect to $\eta$ ). Since $p>1 / 2$, we get

$$
\sum_{n \geq 1}\left|\int_{0}^{t} p(x-y, t-s) \sigma(y, s) \mathbf{1}_{A}(y, s) \mathbf{1}_{\left(t_{n}, t_{n+1}\right]}(s) d s\right| \leq C \sum_{n \geq 1}\left(\sqrt{t_{n+1}}-\sqrt{t_{n}}\right) \leq C .
$$


An analogue of the Lebesgue theorem for stochastic measures implies that the series on the left-hand side of (18) converges, whence we deduce that $p(x-y, t-s) \sigma(y, s)$ is integrable on $\mathbb{R} \times[0, t]$ with respect to $\eta$ by an analogue of the Beppo Levy theorem [3, Theorem 1.10]. Now we derive from Theorem 3.1 that the integral with respect to $\eta$ and the integral in (17) coincide.

Theorem 3.3 implies that

$$
\begin{aligned}
\int_{0}^{t} d s & \int_{\mathbb{R}} p(x-y, t-s) \sigma(y, s) \mathbf{1}_{\left(t_{n-1}, t_{n}\right]}(s) d \mu(y) \\
\quad & \int_{\mathbb{R} \times[0, t]} p(x-y, t-s) \sigma(y, s) \mathbf{1}_{\left(t_{n-1}, t_{n}\right]}(s) d \eta(y, s) .
\end{aligned}
$$

This equality and the integrability of $p(x-y, t-s) \sigma(y, s)$ on $\mathbb{R} \times[0, t]$ with respect to $\eta$ implies that the limit

$$
\begin{aligned}
\underset{t_{n} \uparrow t}{\mathrm{p}-\lim _{0}} & \int_{0}^{t_{n}} d s \int_{\mathbb{R}} p(x-y, t-s) \sigma(y, s) d \mu(y) \\
= & \mathrm{p}-\lim _{t_{n} \uparrow t} \int_{0}^{t_{n}} d \sqrt{t-s} \int_{\mathbb{R}} \exp \left\{-\frac{|x-y|^{2}}{4 a^{2}(t-s)}\right\} \sigma(y, s) d \mu(y)
\end{aligned}
$$

exists in probability.

Now we apply Lemma 4.1 to the function

$$
q(y, s)=\exp \left\{-\frac{|x-y|^{2}}{4 a^{2}(t-s)}\right\} \sigma(y, s) .
$$

Our goal is to estimate the norm of the function $q(\cdot, s)$ in the Besov space. We conclude from equality $a b-c d=(a-c) b+(b-d) c$ that

$$
\begin{aligned}
& |q(y+h, s)-q(y, s)| \\
& \quad \leq C\left|\exp \left\{-\frac{|x-y-h|^{2}}{4 a^{2}(t-s)}\right\}-\exp \left\{-\frac{|x-y|^{2}}{4 a^{2}(t-s)}\right\}\right|+|\sigma(y+h, s)-\sigma(y, s)| .
\end{aligned}
$$

An easy transformation of the Euler-Poisson integral gives us

$$
\begin{aligned}
& \int_{j}^{j+1-h}\left|\exp \left\{-\frac{|x-y-h|^{2}}{4 a^{2}(t-s)}\right\}-\exp \left\{-\frac{|x-y|^{2}}{4 a^{2}(t-s)}\right\}\right|^{2} d y \\
& \quad \leq \int_{\mathbb{R}}\left|\exp \left\{-\frac{|x-y-h|^{2}}{4 a^{2}(t-s)}\right\}-\exp \left\{-\frac{|x-y|^{2}}{4 a^{2}(t-s)}\right\}\right|^{2} d y \\
& \quad=C(t-s)^{1 / 2}\left(1-\exp \left\{-\frac{h^{2}}{8 a^{2}(t-s)}\right\}\right) .
\end{aligned}
$$

Changing the variable $z=r(t-s)^{-1 / 2}$ in the integral on the right-hand side of (15) we obtain

$$
\begin{aligned}
C(t- & s)^{1 / 2} \int_{0}^{1}\left(1-\exp \left\{-\frac{r^{2}}{8 a^{2}(t-s)}\right\}\right) r^{-2 \alpha-1} d r \\
& =C(t-s)^{1 / 2-\alpha} \int_{0}^{(t-s)^{-1 / 2}}\left(1-\exp \left\{-\frac{z^{2}}{8 a^{2}}\right\}\right) z^{-2 \alpha-1} d z .
\end{aligned}
$$

Taking into account Condition 2 for $\sigma$ in (20) implies that

$$
\|q(\cdot, s)\|_{B_{22}^{\alpha}([j, j+1])} \leq C(t-s)^{1 / 2-\alpha}
$$


for an arbitrary $1 / 2<\alpha<\gamma(\sigma)$. Lemma 4.1 with this $\alpha$ yields

$$
\begin{aligned}
\left|\int_{\mathbb{R}} q(y, s) d \mu(y)\right| \leq \sum_{j \in \mathbb{Z}}\left|\int_{(j, j+1]} q(y, s) d \mu(y)\right| & \\
\leq \sum_{j \in \mathbb{Z}}[|q(j, s) \mu((j, j+1])| & \\
+C(t-s)^{1 / 2-\alpha}\left\{\sum_{n \geq 1} \sum_{1 \leq k \leq 2^{n}} 2^{(1-2 \alpha) n}\left|\mu\left(\Delta_{k n}^{(j)}\right)\right|^{2}\right. & \}^{1 / 2}\right] .
\end{aligned}
$$

Repeating again the reasoning concerning the convergence of the sum (7), we prove the bound

$$
\left|\int_{\mathbb{R}} q(y, s) d \mu(y)\right| \leq K(\omega)(t-s)^{1 / 2-\alpha}
$$

for some version of the integral, where $K(\omega)$ is an almost surely finite random variable and where $t, s$, and $\omega$ are arbitrary. Thus the limit in (19) coincides with the iterated integral on the right-hand side of (17).

\section{A Mild SOLUTION IS A WEAK SOLUTION}

We recall some definitions from 7 .

Let $\mathcal{D}$ denote the set of all infinitely differentiable functions $\varphi: \mathbb{R} \rightarrow \mathbb{R}$ with a compact support. The convergence of a sequence of functions in $\mathcal{D}$ is defined in the standard way if the supports are uniformly bounded and all derivatives converge uniformly.

A linear continuous mapping $V: \mathcal{D} \rightarrow L_{0}$ is called a generalized random function. The family of all generalized random functions is denoted by $\mathcal{D}^{\prime}{ }_{r}$.

For an arbitrary process $\xi(t)$, we put

$$
\frac{d}{d t} \xi(t)=\mathrm{p}_{\Delta t \rightarrow 0} \frac{\xi(t+\Delta t)-\xi(t)}{\Delta t}, \quad t>0,
$$

provided the limit exists.

The weak solution of the stochastic heat equation is obtained in [7. It turns out that the solution can be represented in the following form:

$$
\begin{gathered}
\frac{d}{d t}\left(V_{t}, \varphi\right)=a^{2}\left(V_{t}, \Delta_{x} \varphi\right)+\int_{\mathbb{R}} \sigma(x, t) \varphi(x) d \mu(x), \quad t>0, \\
\operatorname{p}-\lim _{t \rightarrow+0}\left(V_{t}, \varphi\right)=\int_{\mathbb{R}} \varphi(x) d \nu(x), \quad \varphi \in \mathcal{D},
\end{gathered}
$$

where $\left(V_{t}\right)_{t>0}$ is an unknown process whose values belong to the set $\mathcal{D}_{r}^{\prime}$ for every $t$ and where $\mu$ and $\nu$ are stochastic measures on Borel subsets of $\mathbb{R}$.

Equation (21) can be treated as a weak form of the first equation of system (13) corresponding to the case of $f=0$.

The following conditions are assumed in this section.

Condition 3. The function $u_{0}(y)=u_{0}(y, \omega): \mathbb{R} \times \Omega \rightarrow \mathbb{R}$ is measurable and bounded for every $\omega$.

Condition 4. For some $K(\omega)>0$ and $\gamma>1 / 6$,

$$
\left|u_{0}\left(y_{1}\right)-u_{0}\left(y_{2}\right)\right| \leq K(\omega)\left|y_{1}-y_{2}\right|^{\gamma} .
$$


A result of [8] claims that there exists a unique (up to equivalence) solution of equation (14) if Conditions 1-4 hold. Moreover there exists a version of such a solution whose trajectories are continuous with respect to $x$. In Theorem 5.1 we consider a continuous version of the solution.

Theorem 5.1. Let $u(x, t)$ be a continuous solution of equation (14) with $f=0$ and let Conditions 1-4 hold. Assume that $|y|^{\rho}$ is integrable with respect to $d \mu(y)$ on $\mathbb{R}$ for some $\rho>1 / 2$. Then the process $\left(V_{t}\right)_{t>0}$ defined by

$$
\left(V_{t}, \varphi\right)=\int_{\mathbb{R}} u(x, t) \varphi(x) d x, \quad \varphi \in \mathcal{D},
$$

satisfies equation (21) and the following initial condition:

$$
\operatorname{p}_{t \rightarrow+0}\left(V_{t}, \varphi\right)=\int_{\mathbb{R}} \varphi(x) u_{0}(x) d x, \quad \varphi \in \mathcal{D} .
$$

Proof. We check equality (21) for $\varphi \in \mathcal{D}$; that is, we check that

$$
\frac{d}{d t} \int_{\mathbb{R}} u(x, t) \varphi(x) d x=a^{2} \int_{\mathbb{R}} u(x, t) \Delta_{x} \varphi(x) d x+\int_{\mathbb{R}} \sigma(x, t) \varphi(x) d \mu(x) .
$$

Using

we easily prove that

$$
\frac{\partial p}{\partial t}-a^{2} \Delta_{x} p=0, \quad t>0
$$

$$
\begin{aligned}
\int_{\mathbb{R}} p(x-y, t-s) \varphi(x) d x & =\varphi(y)+a^{2} \int_{s}^{t} d z \int_{\mathbb{R}} p(x-y, z-s) \Delta_{x} \varphi(x) d x, \\
\varphi & \in \mathcal{D}, \quad t>s, y \in \mathbb{R} .
\end{aligned}
$$

Multiply both sides of (14) by $\varphi \in \mathcal{D}$ and then integrate on $\mathbb{R}$ with respect to $d x$. Since $\varphi$ is finite, $d x$ generates a finite measure.

According to Theorems 3.2 and 3.3 with $d \mathrm{~m}=d x$ one can change the order of integration. (We prove in Appendix A that the function in the stochastic integral satisfies the corresponding Hölder condition, and this allows us to apply Theorems 3.2 and 3.3 .) With the help of (24) we obtain

$$
\begin{aligned}
\int_{\mathbb{R}} u(x, t) \varphi(x) d x & \\
= & \int_{\mathbb{R}} u_{0}(y) d y \int_{\mathbb{R}} p(x-y, t) \varphi(x) d x \\
& +\int_{\mathbb{R}} d \mu(y) \int_{0}^{t} \sigma(y, s) d s \int_{\mathbb{R}} p(x-y, t-s) \varphi(x) d x \\
= & \int_{\mathbb{R}} u_{0}(y) d y\left(\varphi(y)+a^{2} \int_{0}^{t} d z \int_{\mathbb{R}} p(y-x, z) \Delta_{x} \varphi(x) d x\right) \\
& +\int_{\mathbb{R}} d \mu(y) \int_{0}^{t} \sigma(y, s) d s\left(\varphi(y)+a^{2} \int_{s}^{t} d z \int_{\mathbb{R}} p(y-x, z-s) \Delta_{x} \varphi(x) d x\right) .
\end{aligned}
$$

Then we differentiate with respect to $t$. The derivative of the stochastic integral exists in view of an analogue of a standard result on the differentiability with respect to a parameter (see Lemma 5 in [7]). After simple algebra we derive (23) from (24).

The first equality in (25) yields the initial condition. The standard properties of $p(x, t)$ imply that

$$
\int_{\mathbb{R}} p(x-y, t) \varphi(x) d x \rightarrow \varphi(y), \quad t \rightarrow 0+
$$


and that the absolute values of all these integrals do not exceed $\max |\varphi|$. Therefore, for all $\omega \in \Omega$,

$$
\int_{\mathbb{R}} u_{0}(y) d y \int_{\mathbb{R}} p(x-y, t) \varphi(x) d x \rightarrow \int_{\mathbb{R}} u_{0}(y) \varphi(y) d y, \quad t \rightarrow 0+.
$$

An analogue of the dominated convergence theorem implies that

$$
\int_{\mathbb{R}} d \mu(y) \int_{0}^{t} \sigma(y, s) d s \int_{\mathbb{R}} p(x-y, t-s) \varphi(x) d x \stackrel{\mathrm{P}}{\rightarrow} 0, \quad t \rightarrow 0+
$$

(the absolute value of the integrand in the integral with respect to $d \mu$ does not exceed $t \sup |\sigma| \max |\varphi|)$.

\section{Appendix A. HöLDER CONDITION}

We complete the proof of Theorem 5.1 by checking that the function

$$
g(x, y)=\varphi(x) \int_{0}^{t} p(x-y, t-s) \sigma(y, s) d s
$$

satisfies the Hölder condition with respect to the variable $y$ and uniformly in $x$. (Since $\varphi$ is finite, we may assume that $|x| \leq K$ for some $K>0$.)

We use the following elementary bound:

$$
\int_{0}^{t} \frac{1}{r} e^{-B / r} d r \leq\left|\ln \frac{t}{B}\right|+1, \quad t, B>0
$$

Inequality (26) can be proved by differentiating with respect to $t$ and then by substituting the value $B$ for the argument $t$.

Further, for fixed $b>a$ and $K>0$,

$$
\left|\frac{\partial p(x, t)}{\partial x}\right| \leq C t^{-1} \exp \left[-\frac{x^{2}}{4 b^{2} t}\right], \quad|x| \leq K
$$

(also see, for example, (6.13) [12]).

Using (27) and (26) we have

$$
\begin{aligned}
& \int_{0}^{t}\left|p\left(x-y_{1}, t-s\right)-p\left(x-y_{2}, t-s\right)\right| d s \\
& \quad=\int_{0}^{t}\left|\int_{y_{1}}^{y_{2}} \frac{\partial p(x-y, t-s)}{\partial y} d y\right| d s \\
& \quad \leq C \int_{y_{1}}^{y_{2}} d y \int_{0}^{t} \frac{1}{t-s} \exp \left[-\frac{(x-y)^{2}}{4 b^{2}(t-s)}\right] d s \leq C \int_{y_{1}}^{y_{2}}\left(\ln \frac{4 b^{2} t}{|x-y|^{2}}+1\right) d y \\
& \quad \leq C\left|y_{1}-y_{2}\right|+C\left|y_{1}-y_{2}\right|\left(1-\ln \left|y_{1}-y_{2}\right|\right) \leq C\left|y_{1}-y_{2}\right|^{\beta}
\end{aligned}
$$

for all $y_{1}<y_{2}$ and $0<\beta<1$.

The representation

$$
\begin{aligned}
g\left(x, y_{1}\right)-g\left(x, y_{2}\right)= & \varphi(x) \int_{0}^{t}\left(p\left(x-y_{1}, t-s\right)-p\left(x-y_{2}, t-s\right)\right) \sigma(y, s) d s \\
& +\varphi(x) \int_{0}^{t} p\left(x-y_{2}, t-s\right)\left(\sigma\left(y_{1}, s\right)-\sigma\left(y_{2}, s\right)\right) d s
\end{aligned}
$$

and Conditions 1 and 2 imply that

$$
\left|g\left(x, y_{1}\right)-g\left(x, y_{2}\right)\right| \leq C\left|y_{1}-y_{2}\right|^{\gamma(\sigma)} .
$$




\section{BIBLIOGRAPHY}

1. J. Memin, Yu. Mishura, and E. Valkeila, Inequalities for the moments of Wiener integrals with respect to a fractional Brownian motion, Stat. Probab. Letters 27 (2001), no. 2, 197-206. MR 1822771 (2002b:60096)

2. S. Kwapień and W. A. Woycziński, Random Series and Stochastic Integrals: Single and Multiple, Birkhäuser, Boston, 1992. MR1167198 (94k:60074)

3. V. N. Radchenko, Integrals with Respect to Stochastic Measures, Proceedings of Institute of Mathematics, Academy of Sciences of Ukraine, Kiev, vol. 27, 1999. (Russian)

4. V. N. Radchenko, Integrals with respect to stochastic measures and random linear functionals, Teor. Veroyatnost. i Primenen. 36 (1991), no. 3, 594-596; English transl. in Theory Probab. Appl. 36 (1991), no. 3, 621-623. MR.1141138 (93e:60093)

5. V. M. Radchenko, On the product of random and real measures, Teor. Imovir. Mat. Stat. 70 (2004), 144-148; English transl. in Theory Probab. Math. Statist. 70 (2005), 161-166. MR:2110872 (2005h:60146)

6. V. M. Radchenko, Parameter-dependent integrals with general stochastic measures, Teor. Imovir. Mat. Stat. 75 (2006), 140-143; English transl. in Theory Probab. Math. Statist. 75 (2007), 161-165. MR2321189 (2008k:60113)

7. V. N. Radchenko, The heat equation and the wave equation with general stochastic measures, Ukrain. Mat. Zh. 60 (2008), no. 12, 1675-1685; English transl. in Ukrainian Math. J. 60 (2008), no. 12, 1968-1981. MR2523115 (2010d:60117)

8. V. M. Radchenko, Mild solution of the heat equation with a general stochastic measure, Studia Math. 194 (2009), no. 3, 231-251. MR2539554 (2010j:60157)

9. V. N. Radchenko, On a definition of the integral of a random function, Teor. Veroyatnost. i Primenen. 41 (1996), no. 3, 677-682; English transl. in Theory Probab. Appl. 41 (1996), no. 3, 597-601. MR.1450086 (98f:60002)

10. V. N. Radchenko, Sample functions of stochastic measures and Besov spaces, Teor. Veroyatnost. i Primenen. 54 (2009), no. 1, 158-166; English transl. in Theory Probab. Appl. 54 (2010), 160168. MR 2766653

11. S. Kwapień, M. Marcus, and J. Rosiński, Two results on continuity and boundedness of stochastic convolutions, Annales de l'Institut Henri Poincaré (B) Probability and Statistics 42 (2006), no. 5, 553-566. MR2259974 (2007j:60059)

12. A. Friedman, Partial Differential Equations of Parabolic Type, Prentice-Hall, Inc., Englewood Cliffs, N.J., 1964. MR0181836 (31:6062)

Department of Mathematical Analysis, Faculty for Mechanics and Mathematics, National Taras Shevchenko University, Academician Glushkov Avenue 2, Kiev 03127, Ukraine

E-mail address: vradchenko@univ.kiev.ua

Received 5/OCT/2009

Translated by OLEG KLESOV 